\title{
Tratamientos Térmicos y Corrosión en Niebla Salina en acero inoxidable AISI 316L
}

\section{Heat Thermal Treatments and Corrosion Salt Fog Spray Test in AISI 316L Stainless Steel}

Presentación: 06-07/10/2020

\section{Doctorando:}

\section{Mariano N. Inés}

Grupo de Investigación Metalurgia Física, Departamento Metalurgia, Centro de Desarrollo y Tecnología de Materiales (DEYTEMA), Facultad Regional San Nicolás, Universidad Tecnológica Nacional - Argentina.

mines@frsn.utn.edu.ar

\section{Director/a:}

\section{Graciela A. Mansilla}

\section{Resumen}

En este trabajo se evalúa el comportamiento microestructural de un acero inoxidable AISI 316L, tratado térmicamente y sometido a ensayos de corrosión acelerada, durante 96 horas, en cámara de niebla salina según normas ASTM B117 e ISO 9227. Se destaca que este material tratado térmicamente y enfriado en aire fue el que presentó mayor grado de corrosión, hallándose relación directa entre las partículas de carburo de cromo y la presencia de grietas asociadas con los productos de la corrosión.

Palabras clave: Corrosión, Aceros Inoxidables, Carburos, Grietas.

\begin{abstract}
Microstructural behavior of AISI 316L stainless steel, heat treated and then subjected to accelerated corrosion tests, for 96 hours, in a salt spray chamber according to ASTM B117 and ISO 9227 standards is evaluated. It is noted that this material heat treated and cooled in air was the one with the highest degree of corrosion, finding a direct relationship between the chromium carbide particles and the cracks presence associated with corrosion products.
\end{abstract}

Keywords: Corrosion, Stainless Steel, Carbides, Cracks.

\section{Introducción}

Recientemente, la demanda de aceros inoxidables austeníticos ha aumentado debido a su alto rendimiento en diversas aplicaciones industriales. Estos aceros poseen contenidos en cromo superiores al 16\%, que le permite la formación en superficie de una fina capa pasivadora de óxido de cromo $\left(\mathrm{Cr}_{2} \mathrm{O}_{3}\right)$ que los protege de la corrosión. Sin embargo, este comportamiento dependerá de sus elementos de aleación y del tipo de ambiente corrosivo al que se lo exponga en servicio, como por ejemplo durante tiempos prolongados en atmósferas marinas que pueden simularse mediante ensayos en una Cámara de Niebla Salina. Los iones cloruros promueven la ruptura localizada de la capa pasivadora de los aceros inoxidables permitiendo una rápida reacción de corrosión, que frecuentemente se presenta bajo la forma de picado (Rodriguez et al, (2013), Abd Rashid et al, (2012), Afolabi (2011)). 
A su vez, la característica de inoxidable de estos aceros inoxidables disminuye cuando se los expone a tratamientos de sensitización térmica, fundamentalmente por la precipitación de carburos, como por ejemplo $\mathrm{M}_{23} \mathrm{C}_{6} \mathrm{y} \mathrm{M}_{6} \mathrm{C}$, y en especial la formación de fase sigma $(\sigma)$ muy perjudicial en muchas aplicaciones, debido a que fragiliza el material y permite una alta penetración de la corrosión. Estos carburos son sensibles a la captura de hidrógeno originado como consecuencia de reacciones corrosivas que podrían constituirse en sitios de iniciación preferenciales para las grietas inducidas por hidrógeno (Ren et al, (2008), Novak et al, (2010)). Por otra parte, las pequeñas partículas de carburos $\mathrm{M}_{23} \mathrm{C}_{6}$ cercanas a los límites de grano son los principales obstáculos para la migración de los límites de subgrano y el deslizamiento de las dislocaciones móviles y, por lo tanto, generan una contribución muy importante a la resistencia a la fluencia a largo plazo del material.

En este trabajo se evalúa la incidencia de los tratamientos térmicos en relación al comportamiento corrosivo de un acero inoxidable austenítico AISI 316L expuesto a atmósfera salina, a $35^{\circ} \mathrm{C}$ durante $96 \mathrm{~h}$, para emular las condiciones de servicio.

\section{Desarrollo}

Se emplearon probetas planas, rectangulares de 0,6 mm de espesor de acero inoxidable AISI 316L, cuya composición química se detalla en la tabla 1. El material de partida en condición as-received presentó un tamaño de grano austenítico de $35,3 \mu \mathrm{m}$, con presencia escasa de carburos de cromo distribuidos en el interior de los granos y en algunos limites de grano (figura 1a).

\begin{tabular}{|c|c|c|c|c|c|c|}
\hline \%Fe & \%C & \%Si & \%Cr & \%Mn & \%Ni & \%Mo \\
\hline Bal. & 0,032 & 0,65 & 17,2 & 1,50 & 10,7 & 2,57 \\
\hline
\end{tabular}

Tabla 1: composición química del acero AISI 316L

Inicialmente, el material en condición as-received se sometió a tratamiento térmico para lograr una precipitación homogénea de carburos y fases intermetálicas en la microestructura de granos austeníticos. Esta etapa consistió en exponer a un conjunto de muestras a un tratamiento de homogeneización de $900{ }^{\circ} \mathrm{C}$, durante 120 minutos y posterior enfriamiento en agua (muestras denominadas W). Un grupo equivalente se enfrió en aire (muestras A).

Posteriormente con estos materiales sensibilizados, se realizaron ensayos de corrosión acelerada en cámara de niebla salina (DIGIMESS Modelo QSS-108) a todas las muestras: en condición as-received y las tratadas térmicamente $(W$ y $A)$, hasta 96 horas empleando una solución al 5\% NaCl, de acuerdo con las normas ASTM B117 - 07 (2003) e ISO 9227:2006 (2006). Luego, las muestras se limpiaron mecánica- y químicamente según ASTM G1 (1999).

Una vez finalizado el ensayo de corrosión, se realizó una primera caracterización superficial con lupa (Olympus SZ61) y posteriormente se analizaron los cambios microestructurales con un microscopio óptico (Olympus GX51) y microscopio electrónico de barrido (Fei Modelo INSPECT S50) con análisis de dispersivo de energía, EDX.

\section{Resultados y discusión}

Consecuencia de la severidad de los enfriamientos se desarrollaron distintos tipos de carburos de cromo, cuantificación realizada utilizando un software analizador de imágenes. Se promovió un aumento del 1,5\% en la cantidad de carburos para las muestras enfriadas por aire en relación con las enfriadas con agua, como puede apreciarse en las figuras 1b-1c. 


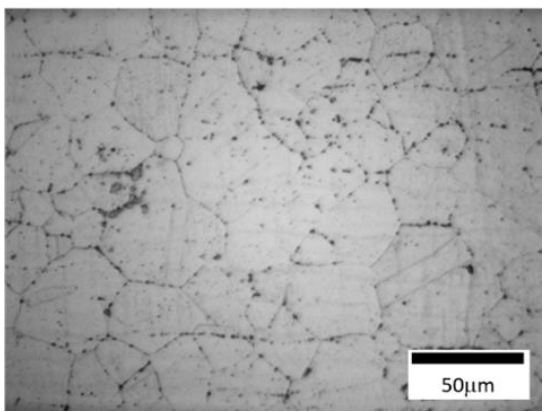

a)

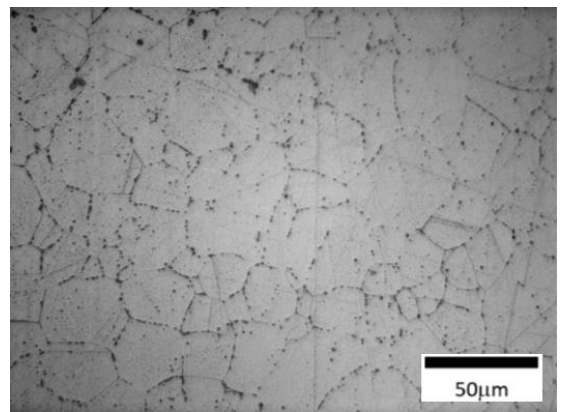

b)

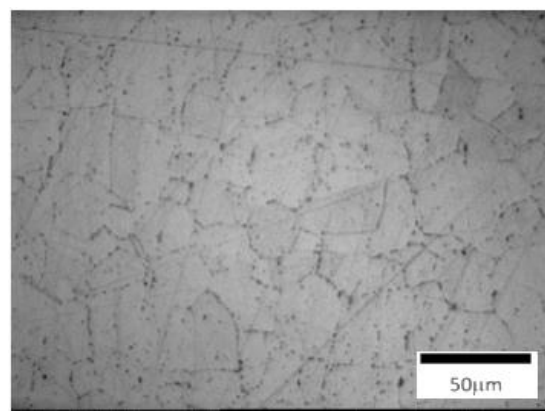

c)

Figura 1: micrografías ópticas de la microestructura del acero AISI 316L, según: a) muestra as-received, b) muestra tratada térmicamente con enfriamiento en aire (muestra A), y c) muestra tratada térmicamente con enfriamiento en agua (muestra W).

Este comportamiento podría atribuirse a que para mayores velocidades de enfriamiento se inhibe el correcto crecimiento de los carburos de cromo en los bordes de grano austenítico (muestras W). Este resultado está en concordancia con lo expresado por Jang et al, (2019) sobre el efecto de las velocidades de enfriamiento sobre los carburos precipitados y la microestructura de un acero reforzado con dispersión de óxido de $9 \mathrm{Cr}-1 \mathrm{Mo}$, donde también el autor agrega que la precipitación de las partículas de carburos se hace más pequeña y se halla más ampliamente dispersa al aumentar la velocidad de enfriamiento. En las condiciones experimentales de este trabajo, las condiciones de enfriamiento propuestas provocaron pequeña influencia en el tamaño de los carburos, ya que para las muestras enfriadas en agua el tamaño medio de los carburos fue de 1,5 $\mu \mathrm{m}$, mientras que se detectaron tamaños medios de 2,5 $\mu \mathrm{m}$ en las muestras enfriadas en aire. En ambos casos, los carburos se generaron únicamente en los límites de los granos, a diferencia de los encontrados en el material as-received que se hallaban también dentro de los granos austeníticos. De acuerdo con Laureys et. al., (2018) en su trabajo acerca del rol de precipitados de titanio y vanadio en la degradación por $\mathrm{H}$ en materiales ferríticos ha demostrado que en ensayos de tracción realizados con muestras previamente cargadas electrolíticamente con hidrógeno, los precipitados de mayor tamaño poseen un rol dominante en la iniciación de las grietas cuando estas se tratan térmicamente y luego enfrían en aire, debido a que las partículas de carburos actúan como fuertes trampas irreversibles para la captura de hidrógeno.

Así mismo, mediante simulación termodinámica realizada con FactSage 7.0, siguiendo los lineamientos planteados por Inés et al, (2017) en su trabajo acerca del efecto de los tratamientos térmicos en la estabilidad de carburos en aceros inoxidables AISI 316 y AISI 446, se determinó que el carburo más estable de acuerdo con las condiciones de ensayo propuesta es el $\mathrm{M}_{23} \mathrm{C}_{6}$. Además, en base a datos obtenidos mediante análisis SEM/EDX se detectó un bajo contenido de cromo en las zonas adyacentes a estos carburos de cromo, localizados en bordes de granos. Este hecho podría atribuirse a una reacción de precipitación compleja que causa zonas empobrecidas de cromo alrededor de los precipitados como predice Rozenak et al, (1986).

Una vez concluido el ensayo de niebla salina, las muestras tratadas térmicamente (explicado previamente) fueron cuidadosamente secadas con aire caliente y analizadas con lupa estereoscópica. Como resultado, en las muestras en condición as-received (es decir, sin tratamiento térmico previo) se reconoció presencia de óxidos superficiales ubicados de manera aislada. En contraste, todas las muestras tratadas térmicamente mostraron, independientemente del medio de enfriamiento empleado, una pátina rojiza de aspecto superficial irregular y muy rugosa asociada a óxidos de hierro, con claros signos de corrosión. Mientras que la muestra A (enfriada en aire) presentó una pátina casi continua con un 99\% de óxidos en superficie, para aquellas con enfriamiento severo en agua la misma resultó cubrir aproximadamente un al 88\% de superficie, donde se notaron regiones libres de óxidos.

El análisis de grietas en niveles superficiales y subsuperficiales se realizó de acuerdo con la metodología propuesta y aplicada por Inés et al, (2015). Los perfiles superficiales de las muestras, analizados mediante microscopía óptica, se presentan en la figura 2, donde claramente se notan los daños microestructurales ocasionados por el ensayo de corrosión. Las muestras tratadas térmicamente (A y W) presentaron mayor cantidad de grietas superficiales y subsuperficiales en comparación con las ensayadas en condición as-received (sin tratamiento previo), sin embargo, estas últimas denotaron un menor grado de ataque debido a la corrosión, hallándose grietas muy cortas (de hasta $4 \mu \mathrm{m}$ ) asociadas con óxidos de 
hierro, figura 2a. Por otro lado, en las muestras tratadas térmicamente se observó mayor daño. Es decir, en la muestra enfriada por agua (W), figura $2 \mathrm{~b}$, se determinaron grietas superficiales intergranulares de hasta $5 \mu \mathrm{m}$ de longitud, con picaduras de corrosión en áreas adyacentes. Sin embargo, en muestras enfriadas en aire y expuestas a la niebla salina, figura 2c, resultó más evidente la penetración de la corrosión, con grietas, que alcanzaron hasta $15 \mu \mathrm{m}$ de longitud, tanto paralelas como perpendiculares a la superficie de la chapa. La presencia de zonas con una marcada depleción de cromo, corroborada mediante análisis EDX, provocaron discontinuidad de la capa protectora natural del acero que lo sensibilizó al ataque del medio salino. Además, se detectaron carburos de cromo en posiciones intergranulares directamente ligados a dichas grietas. De esta forma, y de acuerdo con Inés et al, (2015), se podría afirmar que los productos de la corrosión asociados a grietas generarían tensiones superficiales en las muestras disminuyendo aún más la resistencia a la corrosión. Sin embargo, como fuera expresado previamente, las muestras enfriadas por aire mostraron el mayor ataque en comparación con todas las muestras analizadas. Asimismo, la cinética de iniciación de estas grietas muy probablemente se vea aumentada cuando hay presencia de hidrógeno en estos precipitados y alrededor de los mismos. Por esto mismo es que no debe desconsiderarse que durante las reacciones de corrosión puede generarse hidrógeno que posteriormente ingresa en el material y queda atrapado en distintos tipos de sitios o trampas microestructurales del acero inoxidable. La propagación de las mismas muy probablemente se vea disminuida con la presencia de hidrógeno en trampas del tipo reversibles (es decir, bordes de grano y dislocaciones), Laureys et. al, (2018). Por lo tanto, deberá ajustarse la selección del tipo de carburos precipitados en la microestructura del material para optimizar la resistencia a la degradación inducida por hidrógeno en base a su futuro uso final.

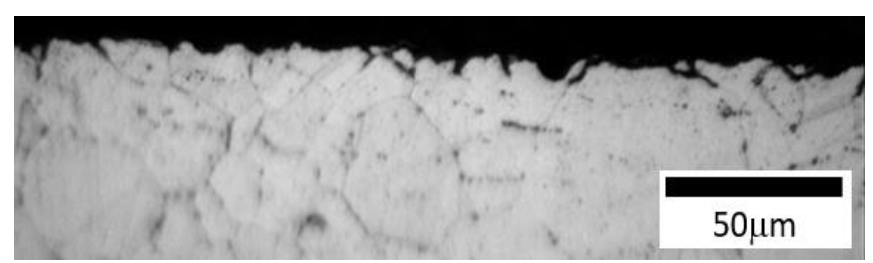

a)

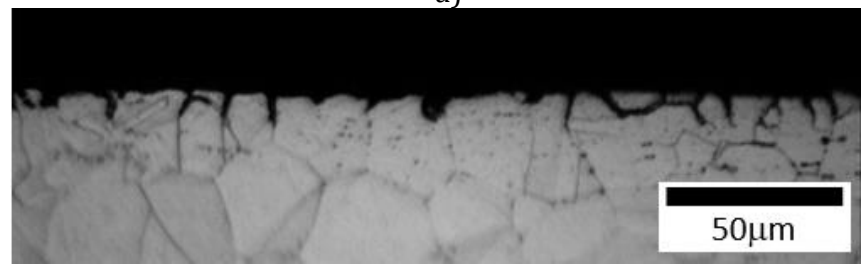

b)

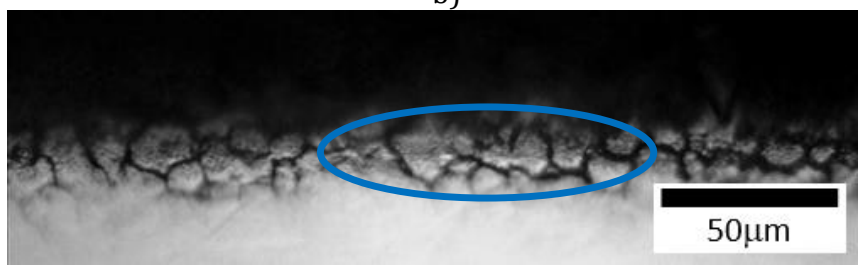

c)

Figura 2: micrografías ópticas donde se aprecia la presencia de grietas en las muestras ensayadas durante 96 horas en niebla salina. Se observa que la degradación del material producto de grietas intergranulares es mayor en la muestra enfriada en aire. Muestra: a) $A R$,

$$
\text { b) W y c) A. }
$$

En este sentido, mediante observación con microscopía electrónica SEM de la muestra enfriada en aire y luego ensayada en niebla salina, pudo corroborarse la presencia de desarrollos masivos de grietas superficiales y subsuperficiales, con extensiones de entre $6 \mu \mathrm{m}$ y $15 \mu \mathrm{m}$ ubicadas preferentemente en los bordes de granos austeníticos, figura 3. Así mismo. se pudo determinar cierta preferencia en el camino seguido por éstas y su vinculación con los carburos, que según análisis EDX realizado, se corresponderían con porcentajes variables de cromo, hierro, manganeso y molibdeno asociado efectivamente a los $\mathrm{M}_{23} \mathrm{C}_{6} \mathrm{y}$ coincidentemente con los cálculos realizados mediante simulación termodinámica, obtenidos como consecuencia de los tratamientos térmicos. 


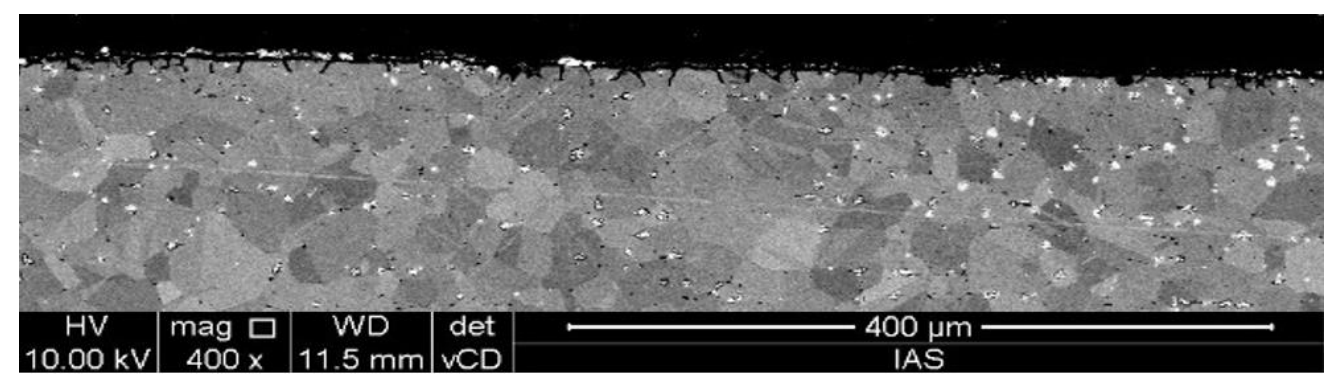

Figura 3: superficies de muestras A, antes y luego de los ensayos de corrosión acelerada. [0.67X]

La precipitación de distintos carburos de cromo en posiciones inter- y transgranulares, sensibilizan al ataque de los iones cloruros, conduciendo a corrosión por picado en los aceros inoxidables al promover la ruptura de la capa pasivadora en áreas localizadas (Inés et al, (2017), Inés et al, (2018)). Esto pudo ser comprobado mediante microscopía electrónica de barrido (SEM) que reveló la presencia generalizada de pits de corrosión, así como de grietas vinculadas con corrosión intergranular.

\section{Conclusiones}

La evidencia experimental obtenida permite establecer que un acero inoxidable austenítico sensibilizado puede sufrir efectos de la corrosión cuando se emplea en ambientes químicamente activos.

Puntualmente sobre la base de los resultados arribados en este trabajo, la menor velocidad de enfriamiento desarrollada por la muestra tratada térmicamente y enfriada en aire presentó mayor grado de avance de la corrosión en un ambiente clorado. Es decir, que la menor cinética de enfriamiento habría promovido un ligero aumento en el tamaño y densidad de los carburos de cromo, dejando mayores áreas con depleción de cromo y favoreciendo una mayor degradación del acero al exponerlo a una atmósfera agresiva.

\section{Referencias}

Abd Rashid, M. W., Gakim, M., Rosli, Z. M. \& Asyadi Azam, M. (2012). Formation of $\mathrm{Cr}_{23} \mathrm{C}_{6}$ During the Sensitization of AISI 304 Stainless Steel and its Effect to Pitting Corrosion. Int. J. Electrochem. Sci., (7), 9465 - 9477.

Afolabi, A. \& Peleowo, N. (2011). Effect of Heat Treatment on Corrosion Behaviour of Austenitic Stainless Steel in Mild Acid Medium. Anales de International Conference on Chemical, Ecology and Environmental Sciences.

ASTM B117 - 07. Standard Practice for Operating Salt Spray (Fog) Apparatus. 100 Barr Harbor Drive, PO Box C700, West Conshohocken, PA 19428-2959, United States. 2003.

ISO 9227:2006 (E). International Standard - Corrosion Test in artificial atmospheres - Salt spray tests. Second Edition. 2006.

ASTM G1. Standard Practice for preparing, Cleaning, and Evaluation Corrosion Test Specimens. PO Box C700, West Conshohocken, PA 19428-2959 USA. 1999.

Inés, M., Asmus, C. \& Mansilla G. (2015). Influence of Total Strain Amplitud on Hydrogen Embrittlement of High Strength Steel, (8), 1039-1046.

Inés, M. \& Mansilla, G. (2017). Efecto de los Tratamientos Térmicos en la Estabilidad de Carburos en Aceros Inoxidables AISI 316 y AISI 446. Anales CONAMET-SAM 2017, Copiapó-Chile.

Inés, M. \& Mansilla, G. (2017). Estudio de la Precipitación de Fases en Aceros Inoxidables y su Interacción con el Hidrógeno. Anales $6^{\circ}$ Encuentro JIM 2017, Buenos Aires, Argentina. 
Inés, M. \& Mansilla, G. (2018). Hydrogen Trapping Sites in AISI 316L and AISI 446 Stainless Steels. Anales of International Conference on Emerging Trends in Materials Science and Nanotechnology 2018, Rome, Italy.

Jang, K. N., Kim, T. K. \& Kim K. T. (2019). The Effect of Cooling Rates on Carbide Precipitate and Microstructure of 9CR1MO Oxide Dispersion Strengthened (ODS) Steel. Nuclear Engineering and Technology, (51), 249-256.

Laureysa, A., Claeysa, L., De Serannoa, T., Depovera, T., Van den Eeckhouta, E., Petrovb, R. \& Verbeken, K. (2018). The Role of Titanium and Vanadium Based Precipitates on Hydrogen Induced Degradation of Ferritic Materials. Materials Characterization, (144), 22-34.

Novak, P., Yuan, R., Somerday, B., Sofronis, P. \& Ritchie, R. (2010). A Statistical, Physicalbased, Micro-Mechanical Model of Hydrogen-Induced Intergranular Fracture in Steel. J. Mech. Phys. Solids, (58), 206-226.

Ren, X.C., Zhou, Q.J., Shan, G.B., Chu, W.Y., Li, J.X., Su, Y.J. \& Qiao, L.J. (2008). A Nucleation Mechanism of Hydrogen Blister in Metals. Metall. Mater. Trans, (39A), 87-97.

Rodríguez, C. J., Figueroa, Y. \& Prin, J. (2013). Efecto de la Temperatura en el Comportamiento del Acero Inoxidable Austenítico 316L frente a la Corrosión Electroquímica. Universidad de Oriente, Venezuela, (25) 3, 2013, 302-308.

Rozenak, P. \& Eliezer, D. (1986). Precipitation Behaviour of Sensitized AISI Type 316 Austenitic Stainless Steel in Hydrogen. Journal of Materials Science, (21), 3065 - 3070. 